\title{
Fast-dm: A free program for efficient diffusion model analysis
}

\author{
ANDREas Voss \\ Albert-Ludwigs-Universität Freiburg, Freiburg, Germany \\ AND \\ JOCHEN VosS \\ University of Warwick, Coventry, England
}

\begin{abstract}
In the present article, a flexible and fast computer program, called fast- $d m$, for diffusion model data analysis is introduced. Fast-dm is free software that can be downloaded from the authors' websites. The program allows estimating all parameters of Ratcliff's (1978) diffusion model from the empirical response time distributions of any binary classification task. Fast-dm is easy to use: it reads input data from simple text files, while program settings are specified by command0s in a control file. With fast-dm, complex models can be fitted, where some parameters may vary between experimental conditions, while other parameters are constrained to be equal across conditions. Detailed directions for use of fast-dm are presented, as well as results from three short simulation studies exemplifying the utility of fast-dm.
\end{abstract}

Diffusion models (Ratcliff, 1978) provide a framework to analyze data from binary decisions. In comparison with simpler approaches (e.g., ANOVAs of response time [RT] means), there are several advantages of this kind of data analysis: First, both RTs and error rates are entered simultaneously in one analysis. This is a great improvement over traditional techniques that are only based on either response times or error rates and thus might lead to misleading results (e.g., about task difficulty) in case of a speed-accuracy trade-off. For example, it might be that the participant's responses slow down not because a task is more difficult but because they set themselves a more conservative response criterion. In this case an analysis based on mean response times would come to wrong conclusions while the effect (adaptation of response strategy) could still be mapped by a diffusion model analysis. Second, the diffusion model is well suited to reflect the structure of the given information. Results are not one-dimensional (i.e., good performance vs. bad performance) but consist of several parameters, which makes it possible to draw detailed conclusions about how a task is performed. In other words, the diffusion model helps to explain why responses are fast or slow and why few or many errors are made. Often, psychological theories allow deriving predictions for individual parameters of the diffusion model (Voss, Rothermund, \& Voss, 2004). Therefore, the diffusion model is a powerful tool to test psychological theories. These structural results are possible because of a third advantage of the diffusion model: The degree of utilization of input data is excellent, because not only RT means are used but the complete RT distribution is analyzed.
On the other hand, there are some problems connected with diffusion model data analysis: First, the computation is expensive. This problem has been recently addressed by Voss and Voss (in press). The authors introduced a new method (the PDE method, based on the partial differential equation describing the diffusion model) that allows fast computations even at high accuracy. The basic idea of the PDE method will be sketched below. If prior distributions for the parameters are available, a Bayesian approach could be used instead (Lee, Fuss, \& Navarro, 2007). A second problem of this kind of modeling is the lack of standard software for diffusion model data analysis. So far, diffusion model analysis was restricted to researchers capable of developing their own solutions. Only recently a MATLAB based program to estimate the parameters of Ratcliff's diffusion model has been introduced by Vandekerckhove and Tuerlinckx (2006). Another solution has been provided by Wagenmakers, van der Maas, and Grasman (2007): Their EZ-diffusion procedure allows to calculate rough estimates for some model's parameter from RT means, standard deviations, and the percentage of errors. However, the EZ model does not cover all parameters, and the utilization of data is not as good as in complete estimation procedures.

In this article, we present a user-friendly, flexible, and efficient program, fast-dm, which addresses both of the mentioned problems. Fast-dm allows considering models where parameters are constrained across conditions or are fixed to predefined values. Fast-dm uses the PDE method (Voss \& Voss, in press) to compute the predicted cumulative

A.Voss, voss@psychologie.uni-freiburg.de 
distribution function (CDF) and it uses the KolmogorovSmirnov statistic (Voss et al., 2004) for the optimization of parameters. The program, including the source code, is available from the fast-dm Web site (www.psychologie .uni-freiburg.de/Members/voss/fast-dm). Fast-dm should be easily portable to most computer systems. It was tested on Linux and Microsoft Windows systems.

In the following sections, we give a short description Ratcliff's (1978) diffusion model and of the PDE method (Voss \& Voss, in press) used in our implementation. Subsequently, details of the fast-dm algorithm and directions of usage are presented, as well as results from simulation studies.

\section{DESCRIPTION OF RATCLIFF'S DIFFUSION MODEL}

The diffusion model is well suited to analyze data from fast binary decisions. It is based on the assumption that information available to the participant is represented as a one-dimensional quantity. A counter on this dimension changes as a function of time and the decisional process continues until the counter reaches either one of two decision thresholds. In the model the dynamic behavior of the counter is described by a Wiener diffusion process. This process is driven by a systematic component and a random component: The systematic component causes a constant change over time, while the random component adds Gaussian noise to the process.

The diffusion process is terminated as soon as it leaves the interval delimited by the decision thresholds and the exit point from the interval represents the binary decision. In other words, a process termination at the lower threshold means that Decision A is reached while a termination at the upper threshold stands for the alternate Decision B. With a given set of parameters, the diffusion model allows to predict RT distributions for both decisions. For a situation in which one decision might be considered correct and the other one wrong, the RT distributions for correct responses and errors, respectively, as well as the ratio of both distributions are thus predicted.

There are several parameters in the diffusion model (e.g., Ratcliff \& Rouder, 1998). In a diffusion model data analysis these parameters are estimated so that the fit between predicted and empiric RT-distributions is optimal. ${ }^{1}$ The result of this parameter estimation procedure can then be interpreted in terms of cognitive processes (Voss et al., 2004). We conclude this section by giving an account of these model parameters. The simple diffusion model consists of four parameters (threshold separation, starting point, drift rate, and response-time constant), which will be described first.

The first parameter is the width of the interval between decision thresholds $(a)$. If $a$ is small, RTs are short and the random influence on the decision process is rather largethat is, many errors occur. On the other hand, large values of $a$ result in fewer error responses and long RTs. The second parameter is the strength (and direction) of the systematic influence on the diffusion process, called the drift rate (v). Large (absolute) values of the drift cause short process duration, both for correct and erroneous responses, and also small percentages of errors, whereas a drift near zero leads to longer process durations and to more similar probabilities of the two process outcomes. The third model parameter regards the starting value of the process $(z)$. The closer the process starts to a threshold, the higher the probability that this threshold is reached due to the random fluctuations. Consequently, if the starting point moves toward one threshold, the percentage of responses connected to this threshold will increase, and RTs of these responses will decrease. The opposite is true, obviously, for the opposite threshold: Here, the process terminates less frequently and exit times increase with increasing distance of starting point and threshold. The fourth parameter covers the duration of all extradecisional parts of the response time. This so-called response time constant $\left(t_{0}\right)$ models the time used by processes of stimulus encoding and, in large parts, the execution of a response.

The full model takes into account that the processing of a task varies to a certain degree across trials of an experiment: intertrial variability is modeled for the starting value $(z)$, the drift rate $(v)$, and the RT constant $\left(t_{0}\right)$. The starting value in each trial is no longer assumed to equal $z$, but instead follows a uniform distribution around $z$ with the width $s_{z}$. Likewise, for the RT constant a uniform distribution with width $s_{t 0}$ around $t_{0}$ is assumed. The drift rate is modeled as a normal distribution with mean $v$ and standard deviation $s_{v}$. A more exhaustive description of the interpretation of the parameters in terms of cognitive processes might be found elsewhere (e.g., Ratcliff \& Tuerlinckx, 2002; Voss et al., 2004).

The degree of noisiness in the model is defined by the diffusion constant (or intratrial variability of the drift). This diffusion constant is a scaling parameter, that is, it cannot be estimated but is set a priori to a fixed value. Fast-dm uses a diffusion constant of one. To compare parameter meter values of fast-dm with solutions that are based on other diffusion constants, all parameters values (but $t_{0}$ ) have to be multiplied by the desired diffusion constant.

\section{THE PDE METHOD FOR DIFFUSION MODEL ANALYSIS}

As pointed out above, diffusion model analysis is computationally expensive. To calculate one predicted RT distribution from a set of parameters, many operations have to be performed, and, while searching the parameter space for an optimal set of parameters, many of these predicted RT distributions have to be calculated. This can easily result in computation times of several hours or even days for a complete analysis. Therefore, choice of an efficient algorithm to compute the required CDFs is of great importance. Recently, Voss and Voss (in press) suggested the PDE method to calculate the CDF for the RT distribution in the diffusion model and showed that processing times were faster by several orders of magnitude, compared to the traditional closed form solution. In the following, we give a very short introduction to the PDE algorithm.

Mathematically, the CDF for a given set of parameters is found by solving a PDE associated with the diffusion pro- 
cess, namely the Kolmogorov backward equation. There is a well-known closed form solution for this problem that allows calculating the CDF as a function of the models parameters (Feller, 1971; see also Ratcliff, 1978; Voss et al., 2004). This formula, however, contains an infinite sum that - at least for certain parameter regions - has to be evaluated many times before it converges satisfactorily. An alternative approach is to solve the PDE numerically (see Voss \& Voss, in press, for details) instead of resorting to the "closed" solution. This method, referred to as the PDE method here, avoids the problem of the infinite sum andsince numerical solutions to PDEs are mathematically well understood - proven standard methods can be employed.

The solution of the Kolmogorov backward equation is the probability that a Brownian motion with constant drift $v$ and start at $z$ hits the boundary $a>z$ before time $t$ and before the first visit at 0 , considered as a function of the time $t$ and the parameter $z$. For the numerical solution, both the $t$ and the $z$ ranges are discretized and the solution is approximated by a function defined on the resulting grid. The algorithm starts at time 0 with a vector containing the initial condition for the different $z$ values on the grid points and then, in each step, uses the approximation for a fixed time to calculate the approximated values at a (slightly) later time. Details of this procedure can be found elsewhere (e.g., Morton \& Mayers, 1994; Press, Teukolsky, \& Vetterling, 1992, chap. 19). Smaller step sizes, in both dimensions, increase the accuracy of the solution but, at the same time, also increase the computational cost.

Besides avoiding the infinite sum of the closed-form solution, the PDE method has the additional advantage that CDFs are computed for the whole range of different starting points simultaneously. Consequently, the best starting point can be picked out directly, thereby reducing the search space by one dimension and increasing the speed of parameter estimation.

\section{SOFTWARE INTERNALS: THE FAST-DM ALGORITHM}

The calculation of the predicted RT distributions in fast-dm uses the PDE method described above. The parameter estimation procedure of fast-dm is based on the Kolmogorov-Smirnov approach (Voss, et al., 2004). That is, the maximal vertical distance of the predicted and the empirical cumulative response-time distribution (i.e., the $\mathrm{KS}$ test statistic) is minimized. To get a single optimization criterion, the response time distributions from both thresholds are merged into one distribution by equipping all reaction times from the lower threshold with negative signs while using positive signs for the times from the upper threshold (Figure 1). The CDF of the resulting combined distribution conserves the original shapes of the individual distributions (as the shapes of the function to the left and to the right of time zero), the error rate is represented as the value of the combined CDF at time 0 .

For many psychological applications some parameters will depend on experimental conditions (e.g., different drift rates are assumed for different stimuli) while the remaining parameters are assumed to be constant over

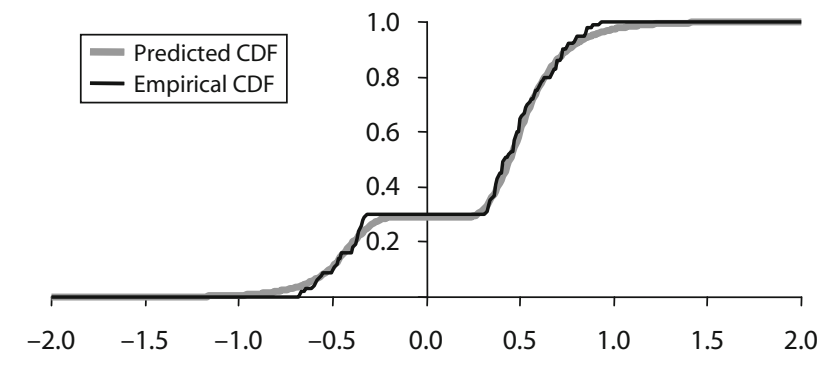

Figure 1. Example for a predicted and an empirical cumulative distribution function (CDF). Fast-dm merges the CDFs of correct responses and errors together by assigning negative signs to all error responses (or, more specifically, to all responses at the lower threshold of the model). Therefore, the value of the CDF at the zero point of the abscissa shows the error rate. The given graph visualizes a data set with about $30 \%$ error responses. The left side of the graph has not the typical shape of an RT distribution because here a mirror image of the original distribution is shown.

conditions. In this case the data set is split according to the experimental conditions and CDFs are calculated and compared for each subset separately, resulting in a different KS value for each condition. When different experimental conditions are used, fast-dm transforms the individual KS values into probabilities values (Conover, 1999; Press et al., 1992) and uses the product of the $p$ values across conditions as the optimization criterion. ${ }^{2}$

To estimate the model parameters a multidimensional search is conducted, using a version of the simplex downhill method (Nelder \& Mead, 1965) to optimize the fit (i.e., to maximize $p$ ). For the parameters $a, v$, and $t_{0}$ the output of the EZ diffusion model (Wagenmakers et al., 2007 ) is used to obtain initial values for the simplex search. For the three intertrial variability parameters, values known as realistic from prior applications are used: In detail, the simplex starts with $s_{z}=0.33 a, s_{v}=0.20, s_{t}=$ 0.15 as the initial values (these values correspond roughly to the medians of the distributions shown in Figure 2 of Wagenmakers et al., 2007). Since the simplex method has sometimes difficulties in high dimensional spaces, the simplex algorithm is consecutively run three times with increasingly strict stop criteria, starting each run with the best solution of the previous run. Our tests indicate that this procedure improves results notably.

In a diffusion model analysis, there is always a speedaccuracy trade-off, that is, the more accurate the calculation, the slower the algorithm runs. There are several points in the program where decisions have to be made about the accuracy settings (e.g., the increment for the calculation of the integrals, and for the discretization parameters of the PDE method). The effects of these settings are not independent of each other: Increasing accuracy at a point in the algorithm that works already well might increase processing time without affecting accuracy because much larger inaccuracies might be caused by another part of the software. While developing fast-dm, a systematic search (reusing the simplex downhill method) was performed to optimize the speed while yielding an a priori defined level of accuracy. This has been done for different accuracy levels. As a result, the accuracy of fast-dm can be controlled 


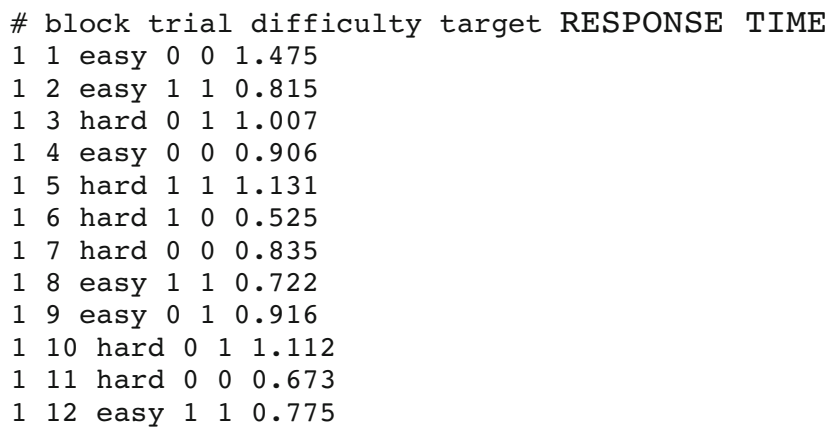

Figure 2. Sample for the first lines of a data file. The first line is ignored by fast-dm because it starts with "\#." Afterward, each line contains the information of one trial of an experiment. For the diffusion model analysis, the first two columns (i.e., "block" and "trial") can be ignored. As can be seen in the example, information about experimental conditions of a trial can either be words or numbers.

by a single precision parameter that internally maps to the optimized parameter values.

\section{USING FAST-DM}

In this article, we describe fast-dm Version 25 . The program, including the source-code, ${ }^{3}$ can be obtained from www.psychologie.uni-freiburg.de/Members/voss/fast-dm. On this site, future versions of fast-dm, and all instructions necessary to install and use these, will be published.

\section{Installation}

To run fast-dm on Linux or Unix systems, download the compressed source-code file (fast-dm-25.zip or fast$\mathrm{dm}-25$.tar.gz, respectively; the contents of theses achieves are identical), save it on your computer and unpack the archive. In the resulting folder "fast-dm-25" type the following commands:

$$
\begin{aligned}
& \text { - / configure } \\
& \text { make }
\end{aligned}
$$

The compiler will automatically build the program fast-dm for the parameter estimation. ${ }^{4}$ If you want to run fast-dm on a Microsoft Windows system, you can either download the precompiled binaries (fast-dm-25-win32.zip) or, if you have a $\mathrm{C}$ compiler, you can also use the source code (see the README file in the source-code archive for some notes on this topic).

\section{Experiment Control Files}

An experiment control file is used to pass commands for a specific experiment to fast-dm. The control file specifies which data files are to be read and what kind of model should be fitted to the data. By convention control files use the file name extension ".ctl" and by default fast-dm tries to read the description for the analyzed experiment from the file "experiment.ctl." On systems supporting command line arguments different experiment control files can be chosen by calling fast-dm with the name of a control file as the first argument.

Control files are evaluated line by line. Empty lines and lines starting with “\#” are ignored. All lines (including the last command line) must be completed by a new-line character (i.e., the "return" or "enter" key has to be used). For all other lines the first word is interpreted as a command, the following words are used as arguments to this command. Table 1 summarizes all possible commands. Most commands are optional and some commands may be used more than once: For example, if several parameters depend on experimental conditions, the command depends has to be repeated for each affected parameter. In the control file, commands must always follow the order as presented in Table 1.

The command precision value defines the accuracy of the calculation of the predicted CDFs. The entered value has to be a real number of 1.0 or above, with accuracy and computation time increasing with higher numbers (values smaller than 2 provide no useful results due to large inaccuracies and values greater than 5 will lead to an unreasonable long processing duration). If the command is not used, the default value of 3.0 is employed.

To set parameters to a fixed value, the command set parameter value has to be applied. Valid entries for $p a-$ rameter are $a, z, v, t_{0}, s_{z}, s_{v}$, and $s_{t 0}$. For value, the desired value of the parameter is entered. For example, to set the variability of the RT constant to zero the command set sto 0 is used. For the starting point the set command works somewhat differently; $z$ is not set to an absolute value but to a fraction of $a$. Consequently, the command set $z \quad 0.5$ fixes $z$ to be equal to $0.5 a$ - that is, the process starts at the midpoint between both thresholds. The set command is optional; if no set command is provided, all parameters are estimated.

The command depends parameter condition can be used to allow parameters to take different values in different conditions of an experiment. For the parameter argument, any parameter name can be inserted (see above for valid parameter names). For condition, a new name has to be defined here. The name can be chosen freely but it must not contain any blanks. For example, the command depends $v$ stimulus means that for each kind of stimulus, which is defined below, another drift rate is estimated. It is also possible that a parameter depends on more than one condition. For example, in a design with two kinds of 
Table 1

Commands of the Control File for a Fast-dm Experiment

\begin{tabular}{|c|c|c|}
\hline Command & Example & Description \\
\hline precision value & precision 2.0 & $\begin{array}{l}\text { Defines the precision of calculation (optional command; default } \\
\text { value is } 3.0 \text {, minimum is } 1.0 \text { ). }\end{array}$ \\
\hline set parameter value & set st0 0 & $\begin{array}{l}\text { A parameter is not estimated but fixed to the given value (optional } \\
\text { command; default is no constraints). }\end{array}$ \\
\hline depends parameter condition & depends v stimulus & $\begin{array}{l}\text { For different experimental conditions, different values for the } \\
\text { parameter are estimated. Asterisks indicate that the corresponding } \\
\text { field is ignored (optional command; by default, parameter values } \\
\text { do not depend on any conditions). }\end{array}$ \\
\hline format RESPONSE TIME... & format * stim RESPONSE TIME & $\begin{array}{l}\text { Defines the format of each line of the data file(s) (required } \\
\text { command). }\end{array}$ \\
\hline load "filename" & load "*.dat" & $\begin{array}{l}\text { The load command defines the names of the data files. Wild- } \\
\text { cards }(*) \text { can be used as templates (required command). }\end{array}$ \\
\hline save "filename" & save "*.out" & $\begin{array}{l}\text { The save command defines the names of individual output files. } \\
\text { Wildcards may also be used in the same way as in the load com- } \\
\text { mand (optional command). }\end{array}$ \\
\hline log "filename" & log "protocol" & $\begin{array}{l}\text { The log command works like the save command; however, here } \\
\text { all results are saved within a common file (optional command). }\end{array}$ \\
\hline
\end{tabular}

stimuli (e.g., target vs. distractor) and two levels of difficulties (e.g., easy vs. hard) the command depends $V$ stimulus difficulty causes four different drift rates to be estimated, one for easy targets, difficult targets, easy distractors and for difficult distractors, respectively. The depends command is optional. By default, no parameter depends on experimental conditions.

The first required command is format. It specifies the composition of the data files (see below). Specifically, it defines the order of information that is read from each line of a data file. All condition names that are used by a depends command have to be specified again in the format definition. In addition, the template RESPONSE has to be used to indicate whether the process outcome of a line of the data file belongs to the lower threshold (value 0 ) or to the upper threshold (value 1), respectively. The template TIME is used for the RT. Asterisks indicate that a column of the data file is ignored for the diffusion model analysis (e.g., the trial number). For example, the command format * * stimulus * difficulty * TIME RESPONSE indicates that the third column of the data file contains the information about the stimulus type, the fifth column contains difficulty information of a trial, the seventh column contains the reaction time in seconds and the eights column contains the actual response.

The command load "filename" is also required. The load command defines the names of one or more data files. File names have to be enclosed in double quotes. An asterisk may be included in the filename as a wildcard. In this case, all matching files within the current directory are load sequentially, and for each data file, a new set of parameters is estimated. For example, load "*. dat " is used to analyze all data files with the extension ".dat." Each data file has to have the same format. If the file name does not include an asterisk, fast-dm loads only one data file.

There are two different ways to save the results of fast-dm. First, it is possible to save results in individual save files - that is, for each data file one save file can be generated, containing the estimated parameter values, the fit index, and the elapsed calculation time. This is done with the save "filename" command. Like in the load command, the use of an asterisk as wildcard is possible; however, load and save commands must match: whenever the load command contains an asterisk, so must save and vice versa. The save command is optional. It can be replaced by the log command (see below).

Since individual log files are not very convenient for further processing in most statistical analysis programs, fast-dm offers the possibility to save all results within one summary log file. This is done by the command $\mathrm{log}$ "filename". The log file name must not contain any wildcards. The log command is optional. However, fast-dm cannot be used without output; therefore, either save or log has to be used.

Whenever there is a conflict between commands (e.g., if depends uses an identifier that is not part of the load command), or between commands and data files (e.g., if there are less columns in the data file than defined in the format statement) an error message is returned, and fast-dm is aborted. Table 2 gives examples for a very simple and for a more complex control file.

\section{Data Files}

Data files contain the participants' responses and measured reaction times. Optionally they can also contain information about experimental conditions. Typically each data file describes one session of one participant in an experiment. By convention data files use the file name extension ".dat."

Data files are evaluated line by line. Empty lines and lines starting with "\#” are ignored. All other lines must contain the entries given by the "format" statement in the control file, in the order defined there and separated by white space.

The values in the Response column must be either 0 (for the lower threshold) or 1 (for the upper threshold). In the experiment, these values will either correspond to the actual response (e.g., left key vs. right key) or to the success value (i.e., correct vs. error). The entries in the Time 
Table 2

Examples of Two Control Files

\begin{tabular}{|c|c|}
\hline Content of "experiment.ctl" & Description \\
\hline $\begin{array}{l}\text { format RESPONSE TIME } \\
\text { load "exp.dat" } \\
\text { save "results.dat" }\end{array}$ & $\begin{array}{l}\text { The file exp.dat is loaded. This text file must contain two values in each line: First the re- } \\
\text { sponse, and second the response time. All parameters }\left(a, z, v, t_{0}, s_{z}, s_{v}, s_{t 0}\right) \text { are estimated from } \\
\text { the data, and results are saved to file results.dat. For the precision, the default value } 3.0 \text { is used. }\end{array}$ \\
\hline $\begin{array}{l}\text { precision } 2.0 \\
\text { set } z 0.5 \\
\text { set st0 } 0 \\
\text { depends a task } \\
\text { depends v stim task } \\
\text { format task * stim RESPONSE TIME } \\
\text { load "*.dat" } \\
\text { save "*.out" } \\
\text { log "protocol.log" }\end{array}$ & $\begin{array}{l}\text { Fast-dm loads all files with the affix ".dat" from the active directory. If a file is found, param- } \\
\text { eters are estimated and saved to corresponding files with the affix ".out," and, additionally, to } \\
\text { the common output file called protocol.log. The starting point is not estimated but fixed to } a / 2 \text {, } \\
\text { and the variability of } t_{0}\left(\text { i.e., } s_{t 0}\right) \text { is not estimated but fixed to } 0 \text {. For each of several different } \\
\text { tasks, separate values for } a \text { are estimated. Likewise, different drift rates are estimated for each } \\
\text { stimulus type within each task. For the sake of speed, the precision is reduced to } 2 \text {. }\end{array}$ \\
\hline
\end{tabular}

column must be floating point numbers, giving the RT in seconds. All other entries correspond to experimental conditions and can be either numbers or words. Figure 2 shows an example of the first lines of a data file. The command format * * difficulty target RESPONSE TIME could be used in the control file to read this data.

\section{Save Files}

Save files are used by fast-dm to store the results of the parameter estimation process. For each data file read a separate save file is written. By convention, data files use the file name extension ".out." Save files are plain text files containing the estimated (or fixed) values for all parameters, the probability value of the KS statistic, and the duration of the estimation procedure in seconds. If the depends command is applied, parameters are indexed; indices are separated by an underline from the parameter name. As indices the number (or word, respectively) defining the experimental condition in the data file is used. If a parameter depends on more than one condition, indices are given in the order of condition specifiers in the depends command.

\section{Summary Log Files}

In summary $\log$ files information from different data files can be accumulated within a single output file. The first line of the summary log file contains the names of the variables. Following this, the results for each data file are saved in a new line. The summary log file is convenient to be read by standard statistical software packages (e.g., $R$, EXCEL, or SPSS) for further interference statistical analysis of the estimates. To get this kind of output the log command has to be used.

\section{Screen Output}

While active, fast-dm gives direct output in a text terminal on the screen (see Figure 3 for an example). In the first four lines, information about the experiment is given. First, the name of the control file is presented. Then, the chosen precision value, and the name (or the template for the name) and format of the data files are shown, as defined by the load command. The fourth line contains a list of all parameters that are optimized. If any parameter depends on an experimental condition, it is indexed with the name of the condition variable.

Starting from the fifth line, information for a specific data set (i.e., one data file) is presented. First, the name of the data file is provided. Then, all experimental conditions are listed: That is, if one or more parameter depends on a condition variable, the data set is divided into subsamples; if no dependencies are defined, only one sample is listed. In any case, all parameters belonging to one specific condition are presented in one line. Parameters are indexed as described in the section $\log$ files. For each condition, the number of both responses ( 0 vs. 1$)$ is specified.

Below, the best fit values ( $p$ values) of three consecutive runs of the SIMPLEX algorithm are shown, as soon as a processing loop is finished. Note that a run of the simplex might take some minutes, especially for high precision values, large samples and many conditions. After the third run of the simplex, the estimates for all parameters are shown.

\section{APPLICATION EXAMPLES}

\section{Application Example 1: Simple Models}

For Example 1, three data samples with 20, 200, and 2,000 reactions, respectively, have been simulated from the same sets of parameter values (see note 2 ). The data sets were saved to the files "sample1.dat" to "sample3.dat." In the control file, the following commands were used:

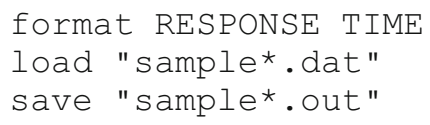

The original values used for the simulation as well as the results of fast-dm are shown in Table 3. As can be seen, the program can recover most parameter values well. Even from only $20 \mathrm{RTs}$, it is possible to get a rough estimate of the true parameter values. It is much more difficult to reestimate the variability parameters. The reason for this difficulty is that they have a rather small influence on the shape of the CDF; nonetheless, including these parameters might in some cases increase model fit considerably (Ratcliff \& Tuerlinckx, 2002). 


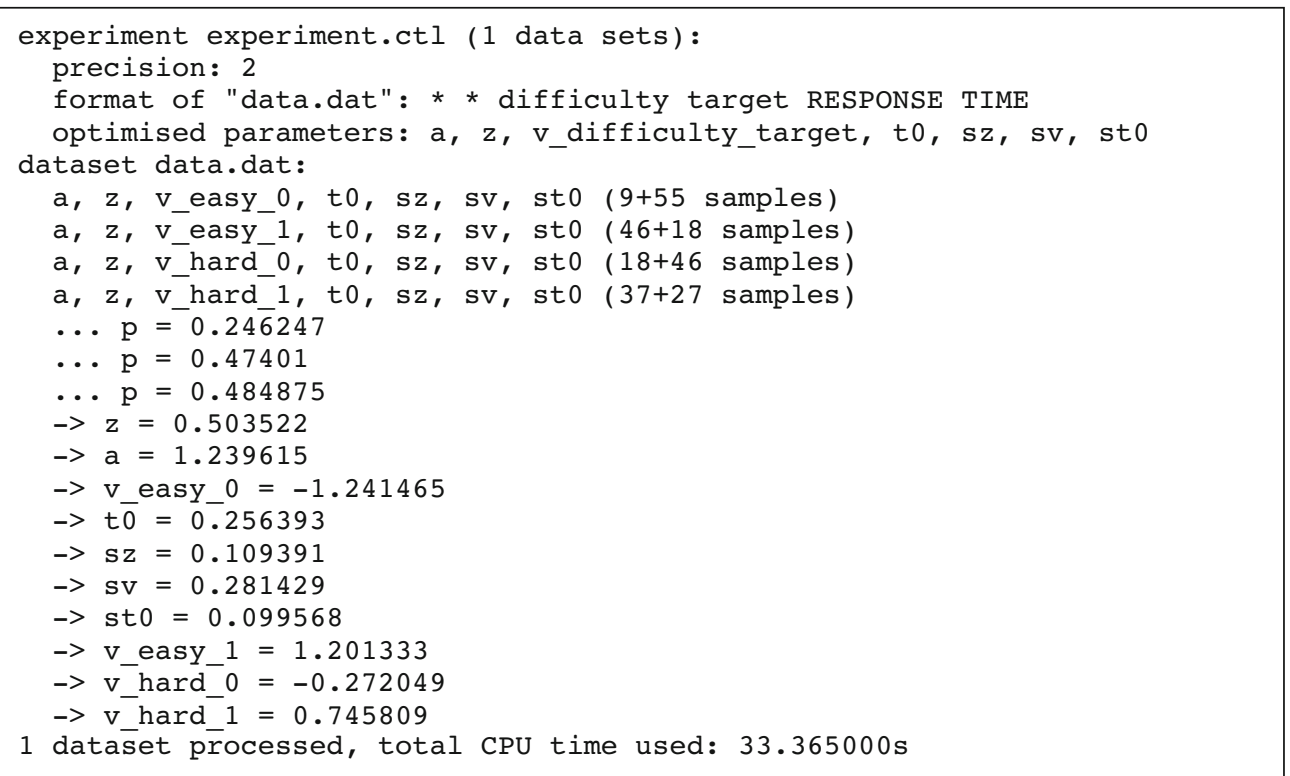

Figure 3. Sample of the screen output of fast-dm. The output contains information about the experiment (Lines 1-4) and about the specific data set being processed (starting from Line 5). Because there are four stimulus types, the data set is divided in four subsamples. For example, in Subsample 1 (containing stimuli of the type "easy_0") there were 55 responses coded with " 0 " and 9 responses coded with " 1 ." The three $p$ values are the results from the three consecutive runs of the simplex algorithm. Below, the estimates for all parameters are shown. Note that stimuli of the target type 0 have a negative drift, because these stimuli require the Alternative Response $B$, which is connected to the lower threshold.

\section{Application Example 2:}

\section{Constraining Parameters to Fixed Values}

Example 2 gives two examples of how to fix certain parameters (Table 4). To analyze Data Set 1, the following control file was used:

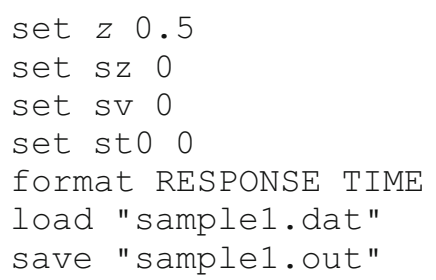

Table 3

Original and Recovered Parameter Values From the Three Data Sets of Application Example 1

\begin{tabular}{lcccc}
\hline & & \multicolumn{3}{c}{ Recovered Values } \\
\cline { 3 - 5 } Parameter & $\begin{array}{c}\text { Original } \\
\text { Values }\end{array}$ & $\begin{array}{c}\text { Data Set 1 } \\
(n=20)\end{array}$ & $\begin{array}{c}\text { Data Set 2 } \\
(n=200)\end{array}$ & $\begin{array}{c}\text { Data Set 3 } \\
(n=2,000)\end{array}$ \\
\hline$a$ & 1.00 & 1.10 & 0.99 & 1.00 \\
$z$ & 0.50 & 0.29 & 0.50 & 0.50 \\
$v$ & 1.00 & 1.74 & 0.87 & 1.04 \\
$t_{0}$ & 0.30 & 0.33 & 0.29 & 0.31 \\
$s_{z}$ & 0.00 & 0.32 & 0.16 & 0.34 \\
$s_{v}$ & 0.00 & 0.15 & 0.44 & 0.40 \\
$s_{t 0}$ & 0.00 & 0.18 & 0.08 & 0.02 \\
$p$ & & 1.00 & 0.99 & 1.00 \\
Time & & 17.58 & 51.57 & 113.63 \\
\hline
\end{tabular}

Note - $p$, probability of the Kolmogorov-Smirnov statistic; Time, duration of the parameter estimation procedure (in seconds).
As can be seen from Table 4, parameters are recovered well, and the computation of this simple model is very fast. For Data Set 2, $z$ was constrained to a specific value that prohibited recovering the original value: Data was simulated for an asymmetric model - that is, a model in which $z=0.8 a$. Nonetheless, for the recovery, $z$ was set to $0.5 a$. This example should illustrate that "wrong" constraints lead to bad statistical fit. The following commands were used:

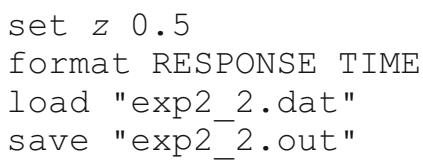

The results show that estimates for the drift and for the variability of the drift are poor. This is due to a trade-off effect: Because the large number of responses at the upper threshold cannot be mapped on $z$, it is mapped on $v$. Nonetheless, the predicted CDF does not fit the data well: The significant $p$ value of the model indicates a model misfit, as was expected.

\section{Application Example 3: \\ Constraining Parameters Across Conditions}

For Example 3, a more complex data set was simulated: Four experimental conditions defined by a $2 \times 2$ design were assumed were $z$ and $v$ depend on conditions whereas all other parameters were assumed to be constant over conditions (Table 5). For each of the four cells of the design, 200 responses were simulated, resulting in a total 
Table 4

Original and Recovered Parameter Values From the Two Data Sets of Application Example 2

\begin{tabular}{cccccc}
\hline & \multicolumn{2}{c}{ Data Set $1(n=200)$} & & \multicolumn{2}{c}{ Data Set $2(n=200)$} \\
\cline { 2 - 3 } \cline { 5 - 6 } Parameter & Original & Recovered & & Original & Recovered \\
\cline { 1 - 3 }$a$ & 1.00 & 1.00 & & 1.00 & 0.81 \\
$z$ & 0.50 & - & & 0.80 & - \\
$v$ & 1.00 & 0.83 & & 0.00 & 2.20 \\
$t_{0}$ & 0.30 & 0.28 & & 0.30 & 0.27 \\
$s_{z}$ & 0.00 & - & & 0.00 & 0.06 \\
$s_{v}$ & 0.00 & - & & 0.00 & 1.55 \\
$s_{t 0}$ & 0.00 & - & & 0.00 & 0.02 \\
$p$ & & .99 & & .02 \\
Time & & 0.17 & & 97.62 \\
\hline
\end{tabular}

Note-In the parameter estimation procedure, $z$ was fixed to $0.5 a$ for both data sets. For Data Set 1 , the variability parameters were fixed to 0 . $p$, probability of the Kolmogorov-Smirnov statistic; Time, duration of the parameter estimation procedure (in seconds).

sample of 800 responses. The following commands were used in the control file:

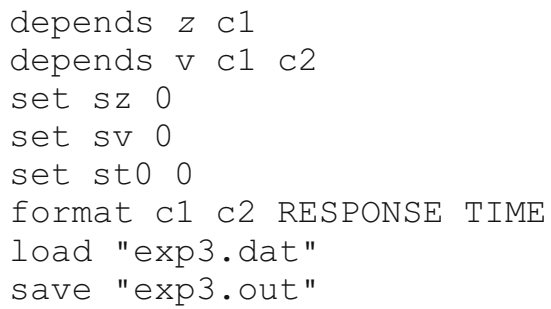

The input consists of one data file (called "exp3.dat") with four data columns: First, there are two variables coding the conditions as in Table 5, followed be the response ( 0 vs. 1 ) and the RT. The depends commands cause a complex model, in which parameters have equal value across conditions besides $z$ (depending on $\mathrm{c} 1$ ) and $v$ (depending on both c1 and c2). Obviously, other models would be possible as well. For example, the simulated data could also be recovered if the drift would only depend on the condition variable $\mathrm{c} 2$. Variability parameters are set to zero. Again, fast-dm could recover most parameter values well (Table 5).

Table 5

Original and Recovered Parameter Values From the Four Data Sets of Application Example 3

\begin{tabular}{|c|c|c|c|c|c|c|c|c|}
\hline & \multicolumn{4}{|c|}{ Original Data Set } & \multicolumn{4}{|c|}{ Recovered Data Set } \\
\hline & 1 & 2 & 3 & 4 & 1 & 2 & 3 & 4 \\
\hline \multicolumn{9}{|c|}{ Condition } \\
\hline $\mathrm{c} 1$ & 1 & 1 & 2 & 2 & 1 & 1 & 2 & 2 \\
\hline $\mathrm{c} 2$ & 1 & 2 & 1 & 2 & 1 & 2 & 1 & 2 \\
\hline \multicolumn{9}{|c|}{ Parameter } \\
\hline$a$ & \multicolumn{4}{|c|}{1.00} & \multicolumn{4}{|c|}{1.00} \\
\hline$z$ & \multicolumn{2}{|c|}{0.70} & \multicolumn{2}{|c|}{0.30} & \multicolumn{2}{|c|}{0.71} & \multicolumn{2}{|c|}{0.30} \\
\hline$v$ & 2.00 & 0.50 & 2.00 & 0.50 & 2.57 & 0.32 & 1.91 & 0.23 \\
\hline$t_{0}$ & \multirow{3}{*}{\multicolumn{4}{|c|}{0.30}} & \multicolumn{4}{|c|}{0.30} \\
\hline$p$ & & & & & \multicolumn{4}{|c|}{0.47} \\
\hline$t$ & & & & & \multicolumn{4}{|c|}{15.89} \\
\hline
\end{tabular}

Note-Each data set consists of 200 responses. $p$, probability of the Kolmogorov-Smirnov statistic; $t$, duration of the parameter estimation procedure (in seconds).

\section{SUMMARY}

Fast-dm is a new and flexible tool that allows for a fast and precise diffusion model data analysis. The program uses the PDE method recently introduced by Voss and Voss (in press) and for the parameter estimation the Kolmogorov-Smirnov statistic is applied (Voss et al., 2004). Fast-dm estimates all parameters of Ratcliff's (1978) diffusion model $\left(a, z, v, t_{0}\right.$, $s_{z}, s_{v}$, and $\left.s_{t 0}\right)$. Also, complex models can be fitted, in which some parameters may be valid for a whole data set, while other parameters are allowed to vary between conditions. It is also possible to fix parameters to given values, thereby making more simple models possible (e.g., by setting the variability parameters to 0 or by fixing $z$ to $a / 2$ ).

Fast-dm is free software and the source code is available at www.psychologie.uni-freiburg.de/Members/voss/ fast-dm. For Microsoft Windows, we provide also executable binaries of fast-dm. The program can be used, redistributed and modified under the terms of the GNU General Public License (see note 2 for details). For decades, the diffusion model could only be fitted by a selected group of insiders. The program introduced in the present article will allow many researchers - and even students - to run their own diffusion model analyses.

\section{AUTHOR NOTE}

The research reported in this article was supported by Grant K1 614/31-1 from the Deutsche Forschungsgemeinschaft to Karl Christoph Klauer. Correspondence concerning this article should be addressed to A. Voss, Institut für Psychologie, Universität Freiburg, D-79085 Freiburg, Germany (e-mail: voss@psychologie.uni-freiburg.de).

\section{REFERENCES}

Conover, W. J. (1999). Practical nonparametric statistics (3rd ed.). New York: Wiley.

Feller, W. (1971). An introduction to probability theory and its applications (2nd ed., Vol. 2). New York: Wiley.

Lee, M. D., Fuss, I. G., \& Navarro, D. J. (2007). A Bayesian approach to diffusion models of decision-making and response time. In B. Schölkopf, J. C. Platt, \& T. Hoffman (Eds.), Advances in neural information processing systems 19 (pp. 809-815). Cambridge, MA: MIT Press.

Morton, K., \& MAYERs, D. (1994). Numerical solution of partial differential equations. Cambridge: Cambridge University Press.

Nelder, J. A., \& MEAD, R. (1965). A simplex method for function minimization. Computer Journal, 7, 308-313.

Press, W. H., Teukolsky, S. A., \& Vetterling, W. T. (1992). Numerical recipes in $C$ (2nd ed.). Cambridge: Cambridge University Press.

RatCliff, R. (1978). A theory of memory retrieval. Psychological Review, 85, 59-108.

Ratcliff, R., \& Rouder, J. N. (1998). Modeling response times for two-choice decisions. Psychological Science, 9, 347-356.

Ratcliff, R., \& Tuerlinckx, F. (2002). Estimating parameters of the diffusion model: Approaching to dealing with contaminant reaction and parameter variability. Psychonomic Bulletin \& Review, 9, 438-481.

VAndekerckhove, J., \& Tuerlinckx, F. (2006). Fitting the Ratcliff diffusion model to experimental data. Manuscript submitted for publication.

Voss, A., Rothermund, K., \& Voss, J. (2004). Interpreting the parameters of the diffusion model: An empirical validation. Memory \& Cognition, 32, 1206-1220.

Voss, A., \& Voss, J. (in press). A fast numerical algorithm for the estimation of diffusion-model parameters. Journal of Mathematical Psychology.

Wagenmakers, E.-J., van der Maas, H. L. J., \& Grasman, R. P. P. P. 
(2007). An EZ-diffusion model for response time and accuracy. Psychonomic Bulletin \& Review, 14, 3-22.

\section{NOTES}

1. Different statistics have been proposed as the optimization criterion (e.g., maximum likelihood or chi-square; see Ratcliff \& Tuerlinckx, 2002, for a comparison). Our own implementation is based on the Kolmogorov-Smirnov statistic (Voss et al., 2004).

2 . With an increasing number of conditions, the resulting $p$ values will obviously converge to zero. To get a value that is easier to interpret, the average probability value within each condition can by computed by raising $p$ to the power of $1 / k$, where $k$ is the number of conditions.

3. Fast-dm is free software; you can redistribute it and/or modify it under the terms of the GNU General Public License as published by the Free Software Foundation, either Version 2 of the license, or (at your option) any later version. Fast-dm is distributed in the hope that it will be useful, but without any warranty; without even the implied warranty of merchantability or fitness for a particular purpose. See the GNU General Public License for more details. When you download the source code, a copy of the GNU General Public License should accompany this program; if not, write to the Free Software Foundation, Inc., 51 Franklin Street, Fifth Floor, Boston, MA 02110-1301.

4. In addition, the programs plot-cdf and make-samples are built. Plotcdf is a small tool to get the function values of the predicted CDF in millisecond steps for a given set of parameters. It was used to generate Figure 1. Make-samples is a program to synthesize data sets for a given set of parameters. This program was used to generate the samples for Experiments 1,2, and 3. These tools are not described in this article, but more information can be obtained from the authors or by inspecting the provided source code.

(Manuscript received November 17, 2006; revision accepted for publication February 24, 2007.) 